ISSN: $1130-3743$

\title{
LA TRANSFORMACIÓN EDUCATIVA Y SOCIAL EN LAS COMUNIDADES DE APRENDIZAJE
}

\author{
The educational and social transformation in the \\ learning communities
}

\section{La transformation éducative et sociale dans les communautés d'apprentissage}

José Luis BarRio DE LA Puente

Universidad Complutense. Facultad de Educación. Departamento de Teoría e Historia de la Educación. C/ Rector Royo Villanova, s/n. 28040 Madrid.

Correo-e: jlbarrio@edu.ucm.es

Fecha de recepción: enero de 2005

Fecha de aceptación definitiva: abril de 2005

BIBLID [(1130-3743) 17, 2005, 129-156]

RESUMEN

En este artículo se analiza la innovación, la calidad, la participación, la organización y la transformación educativa en las comunidades de aprendizaje. Comienza con el análisis general del contexto en el que se desarrollan las comunidades de aprendizaje, describiendo los aspectos más importantes de la sociedad del conocimiento. A continuación se realiza una aproximación a las bases teóricas de las comunidades de aprendizaje, desarrollando el proceso de transformación de un centro educativo en una comunidad de aprendizaje, estando implicados en este proyecto los diferentes colectivos de la comunidad educativa. También se desarrollan los aspectos organizativos de las comunidades de aprendizaje, analizando los trabajos por comisiones, los recursos y los planteamientos institucionales. Finalmente, se describe el funcionamiento de las comisiones mixtas de trabajo que organizan y gestionan los distintos proyectos, y los grupos interactivos que son una metodología fundamental para acelerar el aprendizaje. 
Palabras clave: educación, innovación, calidad, enseñanza, aprendizaje, participación, organización, proyecto, transformación, comunidad, eficacia.

\section{SUMMARY}

This article analyzes the innovation, quality, participation, organization and educational transformation of the learning communities. It begins with a general analysis of context in which the learning communities are developed, describing the most important aspects of the society of knowledge. Next, take place an approximation of the theoretical bases of the learning communities, that develop the transformation process of an educational centre into a learning community, where different members of the learning community are involved. It is also developed organizatives aspects of the learning communities, analysing the work done by the commissions, resources and institutional expositions. Finally, it describes how the mixed working commissions operate, organize and manage all the different projects and the interactive groups, which are an essential element to accelerate the learning.

Key words: education, innovation, quality, teaching, learning, participation, organization, project, transformation, community, efficiency.

\section{SOMMAIRE}

Cet article analyse l'innovation, la qualité, la participation, l'organisation et la transformation éducative dans les communautés d'apprentissage. Il commence avec l'analyse global du contexte dans lequel se développent les communautés d'apprentissage, en décrivant les aspects les plus importants de la société du savoir. Après on réalise une approximation aux bases théoriques des communautés d'apprentissage, étant impliqués dans ce projet les différents collectifs de la communauté éducative. Les aspects organisationnels de la communauté d'apprentissage se développent aussi analysant les travaux par commissions, les recours et les exposés institutionnels. Finalement on décrit le fonctionnement des commissions mixtes de travail qui organisent et gestions les différents projets, et les groupes interactifs qui sont une méthodologie fondamentale pur accélérer l'apprentissage.

Mots clefs: éducation, innovation, qualité, enseignement, apprentissage, participation, organisation, projet, transformation, communauté, efficace. 


\section{INTRODUCCIÓN}

La educación del ciudadano del siglo XXI, además de basarse en los cuatro pilares fundamentales del conocimiento ${ }^{1}$ : aprender a conocer, aprender a hacer, aprender a vivir juntos y aprender a ser (UNESCO, 1996); tendrá como serios retos los planteamientos de la diversidad cultural, la globalización y la sociedad de la información y la comunicación. En las últimas décadas se ha pasado de la concepción de la democratización de la cultura a la democracia cultural. Si el desarrollo de la primera consistía en uproporcionar conocimientos culturales, en hacer participar de los beneficios de la elite cultural", la segunda consiste en "asegurar a cada uno (individuos, grupos o pueblos) los instrumentos para que con libertad, responsabilidad y autonomía puedan desarrollar su vida cultural" (Ander-Egg, 1981, 46-47). Por tanto, se deberán diseñar respuestas alternativas para que la población escolar y la comunidad a la que se pertenece tengan éxito y buen desarrollo personal y comunitario.

Se camina hacia un nuevo concepto de educación, la educación para el desarrollo es la que sustenta y pretende transmitir el desarrollo sostenible. El desarrollo sostenible debe plantear un nuevo desarrollo: biológico, económico, político y cultural $^{2}$. Para desarrollar este nuevo concepto de educación en los centros educativos, es necesario implicar a todos los sectores de la comunidad para que se organicen y unan sus esfuerzos, para formar auténticas comunidades de aprendizaje en las que todos participen, colaboren y aprendan. Así, " la comunidad concreta supone un ámbito de limitada pero posible transformación sociocultural que permite alejarse tanto de las concepciones totalitariamente salvadoras de la humanidad a cualquier precio, como del pesimismo que avala lo establecido" (Flecha, 1990, 87).

Los centros educativos se deberán abrir a la comunidad transformándose en verdaderas comunidades de aprendizaje en las que todos los miembros de los diferentes colectivos que las integran (profesorado, alumnado, familias, instituciones educativas, etc.), puedan aportar sus experiencias, conocimientos y competencias para mejorar la realidad educativa del centro y de su entorno, es decir, la calidad educativa. Las comunidades de aprendizaje son proyectos de transformación educativa, cultural y social de los centros educativos y su entorno, que basados en el aprendizaje dialógico y en una educación participativa de toda la comunidad,

1. Tal como señala el Informe de la Comisión presidida por J. Delors, a la UNESCO en 1996, la educación debe estructurarse en torno a estos cuatro aprendizajes fundamentales, que en el transcurso de la vida, serán para cada persona, en cierto sentido, los pilares del conocimiento: aprender a conocer, es decir, adquirir los instrumentos de la comprensión, aprender a hacer, para poder influir sobre el propio entorno, aprender a vivir juntos, para participar y cooperar con los demás en todas las actividades humanas; por último, aprender a ser, un proceso fundamental que recoge elementos de los tres anteriores.

2. Se puede consultar Colom, A. J.; Pérez, P. M. y VÁzquez, G. (2001) en Calidad de vida, ambiente y educación, 30-31. 
intentarán conseguir una sociedad del conocimiento. En definitiva, se concibe la educación como un factor de transformación social, y el centro educativo y su entorno como un conjunto de instrumentos que puestos al servicio de esa transformación social, convertirán al centro en una comunidad de aprendizaje.

\section{ANÁLISIS DEL CONTEXTO DE LAS COMUNIDADES DE APRENDIZAJE}

Uno de los rasgos más importantes que definen a la sociedad actual es la precariedad económica debido a que los recursos en nuestra sociedad están desigualmente repartidos, es la llamada «sociedad 20/80, en la que el $20 \%$ de la ciudadanía acumula el $80 \%$ de la riqueza, mientras que el $80 \%$ de ciudadanos tienen el $20 \%$ de la riqueza". Este contexto económico tiene mucha relación con el contexto social, llegando a la precariedad social, entendida como la dificultad para establecer redes de relación ciudadana, para participar en las dinámicas sociales cercanas, incrementando la situación de exclusión.

Otro rasgo definitorio por el que está marcada nuestra sociedad ${ }^{3}$ es la implantación de las nuevas tecnologías de la información y la comunicación (TICs). Este concepto no es nuevo, aunque en la actualidad está en pleno desarrollo.

En 1941 ya se utiliza el término "tecnología educativa" que se define como "Cambios en la conducta resultantes de la aplicación en la escuela de materiales como: cine mudo o sonoro, prensa escolar, imagen fija visionada o proyectada en forma de diapositiva o filminas, materiales de museo y láminas, mapas y gráficos" (Monroe, 1941, 1323). La tecnología educativa es una teoría de la comunicación aplicada al aula (UNED, 1993).

Se puede considerar, entonces, la tecnología educativa como un campo disciplinar cuyo objetivo es la optimización de las estrategias de enseñanza, entendiéndola así como una de las posibles funciones de la didáctica. La aplicación de las TICs abre nuevas posibilidades a la enseñanza aplicando nuevos métodos y recursos didácticos, así se puede indicar que: "Los orígenes de la tecnología educativa se encuentran en el espíritu y en las acciones de los visionarios que creyeron que el aprendizaje podría ser mejorado con un abanico de métodos, técnicas y recursos que enriqueciesen el entorno educativo" (Ely, 1992). La Tecnología educativa como método de enseñanza es una

forma sistemática de diseñar, desarrollar y evaluar el proceso total de enseñanzaaprendizaje en términos de objetivos específicos basada en las investigaciones sobre el mecanismo del aprendizaje y la comunicación, que aplicando una

3. Además de la precariedad económica y la implantación de las TICs, es importante también el concepto de globalización económica. Para profundizar sobre este concepto, se puede consultar TriLla, J.; Ayuste, A.; Romaña, I. y Salinas, M. (2001) Educación y calidad de vida. Las cosas y uno mismo, 156-159. 
coordinación de recursos humanos, metodológicos e instrumentales y ambientales conduzcan a una educación más eficaz (Mallas, 1979, 22).

Las características más importantes de la evolución de las nuevas tecnologías son las siguientes: la generalización del uso del ordenador personal en el trabajo y en el hogar, la disponibilidad de redes de comunicación baratas y globales, la posibilidad de acceso instantáneo a grandes fuentes de información y la proporción importante de trabajadores dedicados a las tecnologías anteriores. La implantación de las TICs ha dado lugar a la llamada sociedad de la información y la comunicación (SIC) o sociedad del conocimiento ${ }^{4}$ que caracteriza nuestro contexto social. Las personas que no sepan funcionar en este contexto, en este entorno, quedan potencialmente excluidas y son fácilmente discriminadas. "El conocimiento es una de las claves para la supervivencia y el desarrollo personal y colectivo" (Martínez, 2004a, 5).

Los centros educativos harán todo lo posible para proporcionar a su alumnado las competencias que esta sociedad exige no sólo para adaptarse a ella, sino para afrontar críticamente los retos que plantea, entre los que se pueden destacar los retos informacionales y de participación. Sin embargo, en algunos de los centros educativos el logro de estos objetivos educativos, generalmente, no se alcanza debido a ciertas dificultades, entre las que se pueden señalar las siguientes:

a) La precariedad socioeconómica unida al nivel instructivo de las familias, que se acaba correlacionando con el nivel de instrucción de sus hijos.

b) La falta de recursos en el centro, que dificulta una adecuada inclusión de todo el alumnado, y la infrautilización de los recursos existentes en los centros educativos por parte de la comunidad educativa.

c) La falta de una cultura de la transformación que parta de las dificultades para generar un proceso de cambio y empoderamiento real de la educación.

d) Un planteamiento educativo que intenta garantizar unos mínimos instructivos que no son suficientes para afrontar la sociedad de la información y la comunicación.

e) La acumulación de personas con características diversas en algunos centros educativos, debida a una escasa distribución de las mismas en la red educativa sostenida con fondos públicos (la distribución de la diversidad del alumnado no es equilibrada en todos los centros sostenidos con fondos públicos).

f) La escasa comunicación real y posterior implicación de las familias en la dinámica del centro educativo.

Cuando en un centro educativo se dan las circunstancias anteriores, el alumnado plantea diferentes problemáticas que dificultarán la buena marcha educativa

4. También se utiliza el nombre se sociedad-red para referirse a una modificación sustancial de la sociedad causada por el desarrollo de las TICs. 
y plantearán serios problemas de desarrollo. Si comparamos esta situación con otros centros en los que existen otros contextos socioeconómicos más favorables, con muchas más posibilidades de desarrollo, nos encontramos con una situación injusta. Una comunidad de aprendizaje puede desarrollarse en cualquier contexto socioeducativo, independientemente del nivel socioeconómico más cercano, aunque en los contextos con circunstancias como las mencionadas anteriormente es prioritario pensar en alternativas a los sistemas actuales. El desarrollo de las comunidades de aprendizaje en los centros educativos contribuye a superar las dificultades $^{5}$ anteriormente analizadas.

\section{APROXIMACIÓN A LAS BaSES TEÓRICAS dE LAS COMUNIDADES DE APRENDIZAJE}

En los centros educativos es fundamental trabajar con verdaderos proyectos educativos de innovación y experimentación, para conseguir en el alumnado una educación integral y eficaz. El trabajo con proyectos educativos es fundamental para conseguir una enseñanza eficaz, así se indica que:

La pedagogía del proyecto tiene tres componentes principales: la carga, el campo y las herramientas. La carga determina previamente la tarea que se va a realizar, y esto lo debe hacer de una forma concreta, explícita, nada ambigua, y enérgica. También determina quién va a llevar a cabo el proyecto, si es un individuo o un grupo, y cuándo va a comenzar y finalizar [...]. El campo consiste en la información e ideas que se van a movilizar al ejecutar la carga [...]. Las herramientas consisten en las estrategias intelectuales para proporcionar información e ideas con las que trabajar en la carga [...]. En el nuevo sistema, lo más posible, las herramientas de cada disciplina deberían estar disponibles a mano para ser usadas: dominar la disciplina no consistirá en aprender cómo hacer herramientas, sino en cómo utilizarlas de manera constructiva (McClintock, 1993, 161-162).

Conseguir una educación integral y eficaz en el alumnado es prioritario, para formar ciudadanos del futuro, «a través de la educación integral un alumno forma un juicio integrando su compromiso con la cultura, adquiriendo convicciones, preferencias, valores, explicaciones, formas de entendimiento que usa para definirse a sí mismo y a su mundo" (McClintock, 1993, 118).

Para conocer el funcionamiento de las comunidades de aprendizaje, es necesario analizar su origen, tanto en el ámbito internacional como nacional. También es importante valorar las experiencias educativas más significativas de orientación dialógica y participativa, que han sido pioneras y que han obtenido éxito en los centros educativos en los que se han desarrollado.

5. Para superar estas dificultades en los centros educativos, además de desarrollar las comunidades de aprendizaje, también es imprescindible la alfabetización en las nuevas tecnologías de la información y la comunicación. 


\subsection{Experiencias educativas de orientación dialógica en el ámbito internacional}

A continuación se analizan una serie de experiencias educativas internacionales ${ }^{6}$ que se basan en las prácticas dialógicas y participativas, cada una de ellas desde la realidad social en la que se inserta. Todas estas experiencias educativas se desarrollan en ámbitos que con frecuencia han sido marginados en los sistemas educativos (población en situación de riesgo, personas adultas, minorías étnicas, etc.), y surgen para las desigualdades ante los nuevos retos de la sociedad, lo que las hace doblemente interesantes. Estos grandes programas ofrecen un conjunto global de instrumentos, metodologías específicas y cambios en la organización general de los centros educativos, para trabajar en situaciones de riesgo social con los estudiantes y con la comunidad. Por tanto, los referentes históricos internacionales de los que emanan las justificaciones y los criterios para desarrollar una comunidad de aprendizaje son los siguientes:

a) Paulo Freire y su pedagogía liberadora ${ }^{7}$. La etapa de la educación de adultos la concibe como una auténtica oportunidad para el desarrollo y la emancipación de personas y colectivos en situaciones de precariedad.

b) School Development Program ${ }^{8}(S D P)$. Este Programa de Desarrollo Escolar es uno de los mejores y más antiguos programas educativos desarrollados en Estados Unidos a finales de los años sesenta. Este programa pretende ofrecer un plan para las escuelas con problemática de fracaso escolar, problemas familiares, baja motivación docente y problemas disruptivos; también puede encauzar de manera participativa un conjunto de acciones de desarrollo y transformación, tanto a nivel académico como a nivel de las relaciones afectivas interpersonales. En este programa es fundamental la participación de las familias y de los agentes locales y comunitarios. Una de las características importantes del programa es la implicación de todos los componentes del centro educativo (profesorado, alumnado, familias y personal no docente). Se dirige a toda la comunidad (entorno social, empresas, autoridades educativas, etc.) y no sólo al centro educativo, su objetivo no es sólo el éxito académico sino también el desarrollo global de los alumnos puesto que están íntimamente relacionados. Hay tres principios básicos para la implementación del School Development Program: colaboración, consenso y resolución de problemas (Elboj, 2002).

6. Aparte de las experiencias internacionales analizadas, se pueden consultar otras experiencias interesantes en APPLE y BEANE (1997). Para obtener más información también se puede consultar la siguiente página web: http://www.Creatinglearningcommunities.org/.

7. FreIRE, Paulo (1992). Pedagogía de la esperanza.

8. Para obtener más información sobre este programa, se puede consultar la siguiente página web: http://www.schooldevelopmentprogram.org/. 
c) Accelerated schools Plus 9 . Las escuelas aceleradas Plus son un programa iniciado a partir de 1986 por Henry Levin, profesor de la Universidad de Stanford (Estados Unidos), y director del Centro de Investigación Educativa (CERAS) de esa misma Universidad. Estas escuelas aceleradas plantean un proceso mediante el cual, a través de un diagnóstico de los problemas de la escuela, las familias y el profesorado, pero también el alumnado, priorizan un conjunto reducido de proyectos encaminados a acelerar el aprendizaje escolar. Por tanto, las escuelas aceleradas Plus constituyen un movimiento nacional para la reforma de la escuela, se basa en el proyecto para escuelas aceleradas, planificado para reemplazar la recuperación académica de los estudiantes en riesgo por un enriquecimiento académico, a través de la aceleración del aprendizaje. Estudios de investigación en los años ochenta documentaron una población en aumento de estudiantes que estaban en peligro de fracaso educativo porque carecían de las experiencias en sus casas, familias y comunidades en las que se basa el éxito educativo de la escuela (Levin, 1987, 1988; Natrie1lo, McDill y Pallas, 1990). El cambio en una escuela acelerada es grande y para que se produzca es necesario realizar una buena planificación, y tener también una buena ayuda con apoyo técnico y formativo.

d) Success for $A l l^{10}$. Este programa de éxito escolar para todos y todas empezó en 1987 en Baltimore (Estados Unidos), en una cooperación entre la Universidad John Hopkins y el Departamento de Educación de la ciudad, para trabajar en escuelas con muy bajo rendimiento y muchos problemas de asistencia, conflictos, etc. Su director es el profesor Robert Slavin que, basándose en investigaciones sobre psicología educativa, impulsó esta experiencia. Por tanto, este programa estaba destinado a mejorar el rendimiento escolar y la autoestima positiva sobre todo en el alumnado con especiales dificultades proporcionadas por un entorno económico pobre y por problemas de exclusión social, y especialmente a prevenir el fracaso escolar en este tipo de alumnado.

\subsection{Experiencias educativas de orientación dialógica en el ámbito nacional}

En nuestro país también existen referentes para superar el fracaso escolar desde principios participativos, trabajando en escuelas de diversidad cultural, desarrollando propuestas curriculares inspiradas en la pedagogía crítica y en la perspectiva

9. Para obtener más información sobre este programa, se puede consultar la siguiente página web: http://www.acceleratedschools.net/.

10. Para obtener más información sobre este programa, se puede consultar la siguiente página web: http://www.successforall.net/. 
dialógica del aprendizaje. Estas experiencias que han sido muy útiles y que han impulsado la creación de las comunidades de aprendizaje son las siguientes:

a) El CREA de la Universidad de Barcelona y el Equipo dirigido por Ramón Flecha. En España, en 1995, se inició la andadura de la primera comunidad de aprendizaje, en una escuela de Portugalete (Vizcaya), con la colaboración del CREA $^{11}$, grupo de investigación y acción educativa. De los dos grupos citados, el primero ha impulsado las experiencias de las comunidades de aprendizaje, y el segundo ha sido pionero y primer impulsor de las comunidades de aprendizaje en el País Vasco, Aragón y Cataluña. Las bases sociales son la democracia y la necesidad de igualdad educativa para todos y todas. Se parte de la base de que la responsabilidad del éxito social y escolar del alumnado depende sobre todo de la propia escuela y de su preparación previa.

b) El förum IDEA de la Universidad Autónoma de Barcelona. Posteriormente el fòrum IDEA ${ }^{12}$ se ha dedicado a la investigación y desarrollo de la educación comunitaria y de las personas adultas.

c) Los Centros de Educación de Personas Adultas de La Verneda Sant Martí (Barcelona) y de la Concordia (Sabadell). Son centros pioneros en desarrollar propuestas curriculares basadas en la pedagogía crítica, y en la perspectiva dialógica del proceso de aprendizaje con personas adultas. El proyecto del centro de educación de personas adultas de La Verneda Sant Martí ${ }^{13}$ de Barcelona, es donde se inicia la alternativa educativa de las comunidades de aprendizaje, empezando a soñar con un ideal de escuela en el año $1999^{14}$ y proponiendo nuevas iniciativas para hacerla

11. Para obtener más información, se puede consultar la siguiente página web: http://www. comunidadesdeaprendizaje.net/.

12. Fòrum IDEA. Comunidades de aprendizaje (2002).

13. En 1978 un grupo de vecinos y vecinas del barrio de La Verneda en Barcelona se atrevió a soñar la escuela que querían para su barrio. En poco tiempo habían conseguido esta escuela y se disparó la participación (SÁnCHEz AROCA, 1999). Han pasado más de veinticinco años y la escuela sigue funcionando, se trata de seguir soñando una escuela ideal, para todas las personas del barrio, en la que puedan participar con igualdad. Para obtener más información sobre el proyecto del centro de educación de personas adultas de La Verneda Sant Martí, se puede consultar la siguiente página web: http:// www.edaverneda.org/.

14. El sueño es un proceso participativo en el que todos los colectivos de la comunidad piensan en el ideal de escuela (Martínez, 2004c, 12). Primavera de 1999. Un fin de semana, en una casa de colonias, unas 100 personas discuten sobre el sueño de una escuela. Hay personas que apenas saben leer, otras llevan muchos años en las tertulias dialógicas de literatura universal, otras son colaboradoras voluntarias, está bien el profesorado. Muchas personas van por primera vez a estas jornadas, otras llevan siete u ocho años participando. Durante dos días discuten cómo implicarse más en la transformación del barrio, lograr una sociedad de la información para todos y todas, los grandes objetivos para los siguientes cinco años. A media tarde del sábado, bajan a Barcelona a unirse a una concentración contra las masacres serbias en Kosovo. Después vuelven a trabajar hasta la tarde del día siguiente. Algunas bajan a Barcelona por la noche porque hay obligaciones familiares ineludibles. Otras aparecen por la 
realidad en el año $2001^{15}$. Las características que han marcado la línea educativa teórica y práctica son: la reflexión sobre el aprendizaje dialógico, la lucha por la igualdad en la sociedad de la información y el reconocimiento de la persona adulta como sujeto de su propia educación. Otros principios fundamentales de la escuela son: el diálogo, la aceptación de los conocimientos de todas las personas, la transformación, el aprendizaje científico de las materias, la aportación a partir de la realidad de cada grupo, y la reflexión y decisión sobre la propia vida. Por tanto, se propicia una educación que colabora en la formación de personas libres, democráticas, participativas y solidarias.

Los referentes indicados anteriormente, a lo largo de los últimos años, han proporcionado una gran cantidad de material didáctico, publicaciones, experiencias y proyectos que nos permiten extraer a continuación las ideas fundamentales para impulsar la transformación de un centro educativo en una comunidad de aprendizaje.

\subsection{Referentes y criterios para la creación, análisis y valoración de una comunidad de aprendizaje}

Para guiar e impulsar el proceso de creación de una comunidad de aprendizaje en un centro educativo, es muy importante tener en cuenta los siguientes conceptos: la ética y la justicia, la igualdad de las diferencias, el aprendizaje para todos, el aprendizaje dialógico, la aceleración del aprendizaje, la colaboración y la participación, el empoderamiento y la inteligencia cultural.

a) Ética y justicia. Dentro del contexto educativo la ética y la justicia son categorías imprescindibles en cualquier iniciativa o acción educativa. Todas las acciones educativas donde se desarrollan experiencias de comunidades de aprendizaje se fundamentan en la ética y la justicia.

b) Igualdad de las diferencias. Las diferencias expresan rasgos característicos y en las comunidades de aprendizaje se parte de la idea de que todas las personas están en situación de igualdad a partir de sus rasgos o diferencias. La riqueza de la diferencia es imprescindible para poder funcionar

mañana del domingo porque el trabajo del sábado no les ha dejado ir antes. En la sesión final se exponen todas las aportaciones de los grupos, se hace el resumen por temas y se deciden los que serán prioritarios (ElBOJ y otros, 2002, 57)

15. Primavera de 2002. Unas 120 personas debaten sobre la democracia participativa y la democracia deliberativa. Analizan en pequeños grupos de ocho o diez personas los conceptos básicos sobre la democracia, se exponen experiencias internacionales, se dan ideas de cómo hay que difundir la información para que llegue a cada persona, se proponen nuevas iniciativas para el funcionamiento de sus órganos de decisión, y la implicación de la escuela en el barrio y en la sociedad. Esta vez el público ha cambiado un poco. Hay unas cuantas personas de Pakistán, Marruecos y Guinea-Conakry... (Elboj y otros, 2002, 57). 
adecuadamente como comunidad. En el centro educativo, cada colectivo (profesorado, familias, alumnado, instituciones, etc.), tienen una perspectiva de la formación y una visión diferentes sobre la educación, pero todas importantes. Es necesario asumir esta idea y revalorizar la importancia de la participación y de la contribución de los diferentes colectivos según sus posibilidades.

c) Aprendizaje para todos. Un elemento imprescindible en el proyecto de las comunidades de aprendizaje, es tener altas expectativas que facilitan y favorecen el proceso de aprendizaje. En las comunidades de aprendizaje se creará un entorno en el que todos los colectivos implicados puedan aprender empezando por el alumnado.

d) Aprendizaje dialógico. El aprendizaje dialógico centra la relación educativa entre dos o más personas que, a través del diálogo reflexivo y el intercambio de ideas aprenden, transformando la información intercambiada en conocimiento.

El diálogo es muy importante, "las convicciones deben ser profundas, pero nunca deben imponerse a los demás; a través del diálogo se tratará de convencer con amor; lo contrario sería sectarismo" (Freire, 1977, 68). Así, "el encuentro educativo... (incluirá) una acción que trate de cambiar las estructuras en las que se produce el aprendizaje... (esto) supone una relación recíproca entre autorreflexión y acción" (Grundy, 1987, 32).

El análisis de la realidad, los contenidos del currículum, las estrategias, etc., se llevan a la práctica cuando las personas implicadas han participado y dialogado sobre los mismos, surgiendo una cultura compartida por todos los integrantes.

e) Aceleración del aprendizaje. La aceleración del aprendizaje se relaciona con el enriquecimiento del entorno de aprendizaje, y se pretende que éste alcance niveles considerados satisfactorios. Las escuelas aceleradas fueron diseñadas para conducir a todos los estudiantes a la corriente dominante académica a través del enriquecimiento y aceleración académicas, sustituyendo la recuperación por enseñanza con talento y de talento (Levin, 1987). En esta misma línea se puede decir que "la aceleración del aprendizaje tiene que ver con el enriquecimiento del entorno de aprendizaje para que éste se produzca con más celeridad" (Martínez, 2004b, 9). También "la aceleración del aprendizaje se entiende como una medida pedagógica que responde a criterios de justicia y equidad" (Ferrer, 2004b, 21). Así, la aceleración del aprendizaje se deberá adecuar de una forma especial a los contextos desfavorecidos. En los contextos de exclusión social existen las mismas capacidades que en los contextos más favorecidos, pero con estas capacidades no se consiguen objetivos satisfactorios debido a las dificultades existentes en el contexto familiar, comunitario y escolar; estas dificultades se deben superar. 
f) Colaboración y participación. Las comunidades de aprendizaje suponen un incremento muy notable de la colaboración participativa. La participación se puede considerar como un principio educativo fundamental. La participación facilita la integración en el grupo de todos sus miembros, así como la responsabilidad y el compromiso en la realización de todas las tareas. Con la participación se tiene la oportunidad de dialogar, reflexionar y deliberar para tomar por consenso las decisiones más adecuadas, evitando la exclusión. La colaboración y participación de todos los colectivos implicados en la comunidad es fundamental para que se desarrolle con eficacia el proceso de aprendizaje. El profesorado debe cumplir con su función; las familias, el alumnado y demás instituciones del entorno (asociaciones vecinales, grupos culturales, administración local, servicios educativos, etc.), deben colaborar activamente con el proyecto.

g) Empoderamiento. Es el proceso mediante el cual un colectivo toma conciencia de su realidad y desarrolla actuaciones de control de su situación encaminadas a la transformación de esa realidad. En general, se empieza con la toma de conciencia de la realidad discriminatoria y se inicia un proceso de trabajo para eliminar la discriminación, y poder llegar al máximo desarrollo personal y colectivo.

h) Inteligencia cultural. La inteligencia cultural es una de las aportaciones teóricas nucleares en los procesos de transformación comunitarios que se concretan en las comunidades de aprendizaje. La inteligencia cultural es un patrimonio que todos los grupos poseen por el hecho de interactuar entre sí en un determinado contexto.

El concepto de inteligencia cultural desarrollada por el CREA (1995-1998), está basado en las experiencias prácticas en escuelas y en teorías críticas como la de la acción comunitaria de Habermas. Esta inteligencia que supone una interacción donde diferentes personas entablan comunicación y consiguen llegar a entendimientos en los ámbitos cognitivo, ético, estético y afectivo. Así, la inteligencia cultural contempla la pluralidad de dimensiones de la interacción humana y es la base del diálogo igualitario en el aprendizaje y en las relaciones sociales (Elboj, 2002, 98).

Esta inteligencia se utiliza para solucionar problemas planteados, analizar la realidad y construir conocimiento más allá de las fórmulas tradicionales y mecanismos convencionales.

Todos los conceptos analizados anteriormente constituyen referentes y criterios para potenciar la transformación de un centro educativo en una comunidad de aprendizaje, además de favorecer el análisis y la valoración, para posteriormente poder tomar decisiones dirigidas a mejorar el aprendizaje en contextos educativos. 


\section{TRANSFORMaCión DE LA EDUCACión EN UNa COMUNidAD DE APRENDIZAJE}

\subsection{La transformación educativa}

El centro educativo, para conseguir una educación de calidad, debe abrirse a la comunidad educativa y, por tanto, a su entorno próximo, debido a la relación existente entre el desarrollo cognitivo y el entorno sociocultural, siendo evidente que el entorno no condiciona la capacidad sino el proceso y las concreciones del aprendizaje. El desarrollo cognitivo estará influido por el cambio del entorno social y cultural.

Este sentido transformador lo refleja Vigotski diciendo

Al mismo tiempo que admite la influencia de la naturaleza sobre el hombre, la aproximación dialéctica postula que el hombre, a su vez, modifica la naturaleza y crea, mediante los cambios que provoca en ella, nuevas condiciones naturales para su existencia. Esta posición es la clave de nuestra aproximación al estudio e interpretación de las funciones psicológicas superiores del hombre y sirve de base para los nuevos métodos de experimentación y análisis por los que abogamos [...]. Mis colaboradores y yo creemos que el comportamiento humano llega a alcanzar esta "reacción transformadora respecto a la naturaleza" que Engels atribuía a las herramientas. Entonces debemos buscar los métodos adecuados a nuestra concepción (Vigotski, 1979, 98-99).

Para la transformación de un centro educativo en una comunidad de aprendizaje, el punto de partida es:

Una escuela ordinaria con los problemas habituales de este tipo de establecimientos. La problemática afecta al alumnado (fracaso escolar, problemas disruptivos y absentismo), al profesorado (desmotivación y baja autoestima profesional) y a las familias (poca implicación y conocimiento de la dinámica escolar). También el entorno permanece, en la situación inicial, bastante ajeno a la escuela (Martínez, 2004c, 11).

Por tanto, en la transformación ${ }^{16}$ de un centro educativo ordinario en una comunidad de aprendizaje, todos los colectivos y personas que forman parte de la

16. Todos los colectivos implicados en el proceso de transformación definen y acuerdan los aspectos más importantes de la transformación del centro educativo en una comunidad de aprendizaje. Decisiones que irán concretando las diferentes fases del proyecto. Una comunidad de aprendizaje relata su sueño con las siguientes palabras: “Todos los implicados soñaron mucho. De la participación de padres y madres surgieron propuestas sobre cómo contar con ellos en el trabajo educativo. [...] El alumnado en unos casos soñó por escrito, en otros casos mediante dibujos o en conversaciones con los maestros. [...] Todos los sueños de los alumnos y alumnas estuvieron expuestos un tiempo en el vestíbulo. [...] Sus sueños fueron muy diversos, desde tener piscina o animales en el centro, pasando por un aula de informática. Los sueños de los profesores fueron muy amplios como implicar a las familias o los sueños relacionados con los aspectos didácticos" (JAUSs y LunA, 1998, 39). 
comunidad escolar deben vincularse en un proyecto común. Este proceso por el cual un centro educativo ordinario se puede transformar en una comunidad de aprendizaje es un proceso muy complejo y constituye un proyecto importante que se inicia con la concurrencia, participación e implicación de los tres colectivos fundamentales de la comunidad educativa que son: el profesorado, las familias y el alumnado. Así los tres colectivos podrían aportar perspectivas y puntos de vista diferentes contribuyendo al mutuo enriquecimiento personal en dicho proceso.

El desarrollo de una comunidad de aprendizaje en un centro educativo se potenciará teniendo en cuenta los siguientes factores: el asesoramiento en la formación del profesorado por parte de los agentes externos al centro, la participación de las familias, los servicios sociales municipales, los servicios educativos de la zona, los IES (Institutos de Educación Secundaria) del entorno, los voluntariados universitarios y preuniversitarios, y las asociaciones del barrio.

\subsection{Desarrollo de una comunidad de aprendizaje}

Para convertir un centro educativo ordinario en comunidad de aprendizaje, es necesario realizar una planificación destinada a que todos los colectivos implicados conozcan los objetivos y se comprometan con su realización. Las fases necesarias para que se produzca el desarrollo comunitario en un centro educativo son las siguientes: sensibilización, toma de decisiones, sueño, priorización, planificación, implementación y evaluación.

a) Fase de sensibilización. El objetivo de esta fase inicial es conocer el proyecto de transformación educativa, analizar sociológicamente la realidad y la identificación de las principales características, problemas y retos que la sociedad actual plantea a la educación. En la fase de sensibilización se analiza la situación del centro educativo, los problemas, las oportunidades que plantea, las implicaciones en este proceso y la planificación general del proyecto. También se abordan cuestiones importantes como la explicación general del proyecto, las fases y sistemas de aplicación, el análisis de la sociedad de la información, la aplicación en la zona y las consecuencias educativas, el análisis de la transformación del centro en comunidad de aprendizaje, las situaciones de desigualdad educativa, los resultados de los modelos alternativos y el análisis de las necesidades de formación (Elboj y otros, 2002).

b) Fase de toma de decisiones. En esta fase, la comunidad escolar decide la transformación del centro educativo en comunidad de aprendizaje. Son necesarios la máxima participación y compromiso de todo el claustro de profesores, es decir, el 90\% del claustro debe adherirse al proyecto y, por tanto, participar en la experiencia de transformación educativa. El proyecto de transformación educativa iniciará su implementación, una vez 
que todos los colectivos han tomado la decisión formal de participar e implicarse en dicho proyecto.

c) Fase del sueño. A partir de la sensibilización y la toma de decisiones, se llega a la fase del sueño que es un proceso participativo de todos los colectivos implicados en la comunidad, encaminado a "soñar" ${ }^{17}$ el ideal de centro educativo, el que se desearía para un centro educativo, olvidando contextos limitadores. En esta fase se desarrollan un conjunto de actividades y oportunidades para que profesores, alumnos y familias, y representantes del entorno implicados puedan sugerir cómo sería el centro educativo ideal. Los profesores, mediante reuniones y procesos grupales creativos, analizan los aspectos más importantes de calidad educativa en un centro. Las familias van ideando la escuela que sueñan para sus hijos e hijas y también para las propias familias. El alumnado también reflexionará sobre cómo le gustaría que fuese la escuela. Los demás colectivos implicados analizarán lo que es una escuela de calidad, abierta y participativa, en la que se puedan desarrollar actividades de dinamización sociocultural y comunitaria. Al final, después que cada colectivo ha puesto en común las líneas generales de su sueño, se llega a un consenso mediante el diálogo y el razonamiento buscando la mejor educación para todos, y contextualizándolo a las circunstancias de la escuela.

d) Fase de selección de prioridades. Partiendo de las necesidades, el sueño y la realidad, se seleccionarán y priorizarán las actuaciones más necesarias, interesantes e ilusionantes a seguir en el proceso transformador. Los profesores aportan ideas sobre las características internas del centro, las dinámicas, las metodologías y las estrategias para llevar al aula. Las familias y el alumnado aportan su visión particular para dar una idea más completa, y los demás colectivos de la comunidad aportan su visión complementaria. Una vez que se aportan todas las ideas se realiza una selección de las que se van a llevar a la práctica a corto, medio y largo plazo.

e) Fase de planificación. Una vez que se han seleccionado las ideas del proyecto a desarrollar, se puede planificar y organizar, para convertir los sueños en realidades. Cada proyecto se concreta en unos objetivos, contenidos, metodología, actividades, recursos, temporalización y evaluación. En todos los proyectos participan miembros de todos los colectivos participantes. Para la implementación de estos proyectos es imprescindible la participación de toda la comunidad.

17. El momento de decidir iniciar el proceso y toda la fase del sueño son una descarga de energía. A partir de este momento, aunque todos y todas son conscientes del hecho de que el proceso sólo se ha iniciado, nada vuelve a ser como antes, participan todos los colectivos implicados, se realizan cambios, una parte del sueño se ha hecho realidad (ELBOJ, 2002). 
El proceso de transformación en comunidad de aprendizaje es un proceso que busca continuamente la mejora en el aprendizaje. Las fases anteriores sirven para ponerlo en marcha, las posteriores son más bien orientaciones generales integradas para la consolidación del proceso. Para la consolidación del proyecto es importante una continuidad en los equipos de un curso escolar para otro.

f) Fase de implementación del proyecto. Es el desarrollo de los proyectos planificados, en esta fase el centro educativo funciona ya como una comunidad de aprendizaje. El centro está abierto más horas que las ordinarias, en algunas aulas hay más personas adultas que profesorado, se desarrollan actividades al final del horario lectivo en las que el alumnado y las familias pueden participar. En este desarrollo es importante articular muy bien el funcionamiento de las comisiones de trabajo, una para cada proyecto, y la participación e implicación de todos los colectivos que forman parte de la comunidad. La comisión gestora coordina todos los proyectos del centro. Es muy importante el compromiso, la responsabilidad y la crítica de todos los agentes que participan en la comunidad.

g) Fase de evaluación. Por evaluación se entiende la valoración permanente del proceso de transformación del centro educativo en comunidad de aprendizaje con el objetivo de tomar decisiones sobre el proceso educativo para mejorarlo. En este proceso se recoge todo tipo de información para analizarla y valorarla, obteniendo interesantes resultados que servirán para tomar las decisiones pertinentes. En la evaluación ${ }^{18}$ participan todas las personas implicadas en el proyecto, desde el profesorado, las familias y el alumnado. Con la evaluación se pretende mejorar las prácticas del proyecto y animar a los participantes a seguir transformando su centro educativo. Las decisiones tomadas después de la evaluación serán muy importantes para la acción de mejora que permitirá actualizar los planteamientos de la comunidad de aprendizaje. En la evaluación del proceso, no sólo se deben destacar los cambios importantes que hay que introducir, sino también considerar positivas las transformaciones conseguidas hasta el momento.

18. En el proceso de la evaluación también se realiza una investigación en la medida en que se desarrollan actividades y métodos para la mejora de los aprendizajes. Esta investigación comunicativa, con la experiencia del cambio, se puede modificar a partir del análisis y la reflexión realizada. La investigación educativa que se desarrolla, se dirige a la propia comunidad, en la propia comunidad y por la propia comunidad; y permite resolver los problemas, las dudas y las preocupaciones de cualquier miembro de la comunidad a partir de su implicación directa en la acción investigadora. El proceso de transformación de una escuela en una comunidad de aprendizaje, se convierte en un aliciente para una formación mejor del profesorado, de las familias y de las demás personas adultas participantes en el proyecto, lo que implica iniciar procesos formativos. También es necesaria, por tanto, una formación adecuada, debido a que todo cambio necesita instrumentos nuevos y éstos frecuentemente exigen una formación diferente a la que se ha tenido hasta ese momento. 
Las mejores prácticas educativas del mundo y las actuales teorías sociales demuestran que sólo con la interacción comunicativa de las familias, el profesorado, el barrio y la sociedad se puede construir un proyecto educativo útil. Las comunidades de aprendizaje combaten el fracaso escolar, fomentando la convivencia y la solidaridad.

\section{LOS ELEMENTOS ORGANIZATIVOS EN UNA COMUNIDAD DE APRENDIZAJE}

Las comunidades de aprendizaje potencian aspectos como la participación, la implicación y la colaboración de todos los miembros de la comunidad educativa, creando un sistema de organización que responda o se adapte a las necesidades educativas de la sociedad del conocimiento, fundamentalmente a las nuevas tecnologías de la información y de la comunicación.

Las líneas fundamentales y complementarias de actuación que dan sentido a la comunidad, consiguiendo una nueva apertura del centro educativo a la comunidad y una nueva transformación social son: el trabajo por comisiones, la participación y la toma de decisiones.

1. El trabajo por comisiones. Es una forma de organización con un marcado carácter horizontal que permite la participación en la toma de decisiones, de manera directa, a miembros que tradicionalmente eran representados por órganos colegiados como el consejo escolar del centro.

2. La participación. Se planifica de forma transversal, desde los planteamientos institucionales: Proyecto Educativo del Centro (PEC), Proyecto Curricular del Centro (PCC), Documento de Organización del Centro (DOC), Programación General Anual (PGA) y Reglamento de Régimen Interior (RRI), hasta el sistema relacional (trabajo en equipo, reuniones, comisiones y coordinaciones).

3. Toma de decisiones. Se llega al consenso a través de las estructuras de organización (comisiones) y los procesos (participación comunitaria), basándose en los principios teóricos de la formación y los procesos dialógicos (Freire) y los planteamientos de pretensiones de validez (Habermas).

\subsection{Estructura organizativa}

Las comunidades de aprendizaje se organizan por comisiones mixtas de trabajo que responden a las necesidades del centro. Las comunidades de aprendizaje contemplan la creación de un órgano director que se denomina comisión gestora, y se encarga de dirigir y coordinar el programa de proyectos a tenor de los objetivos planteados (Gráfico n.ำ 1). 
a) El trabajo por comisiones mixtas

Estas comisiones están formadas por todos los miembros que quieran participar en las mismas, sin ningún tipo de exclusión y su composición es mixta. Cada comisión está representada por el mayor número de colectivos posible (profesores, alumnos, familias, centros de apoyo al profesorado, ayuntamientos, servicios sociales, centros de salud y otras instituciones educativas).

La estructura de trabajo por comisiones se plantea como una red de comisiones que intercambian información y actúan de forma coordinada a través de una comisión gestora en la cual están todas representadas. La asamblea orienta y legitima todas las acciones que se llevarán a cabo en la comunidad de aprendizaje, a través de las comisiones de trabajo.

Todas las acciones que se llevarán a cabo en la comunidad de aprendizaje, a través de las comisiones de trabajo, son primero orientadas y después legitimadas por la asamblea, que se reúne aproximadamente tres veces al año para establecer, priorizar y hacer un seguimiento del funcionamiento de la comunidad. La asamblea está compuesta por todas las personas que participan de la comunidad de aprendizaje sin excepción y es un órgano legitimador basado en la democracia directa y en el consenso (Martínez Ortiz, 2004, 16).

\section{GRÁFICO n..$^{\circ} 1$ \\ ESTRUCTURA ORGANIZATIVA DE LAS COMUNIDADES DE APRENDIZAJE}

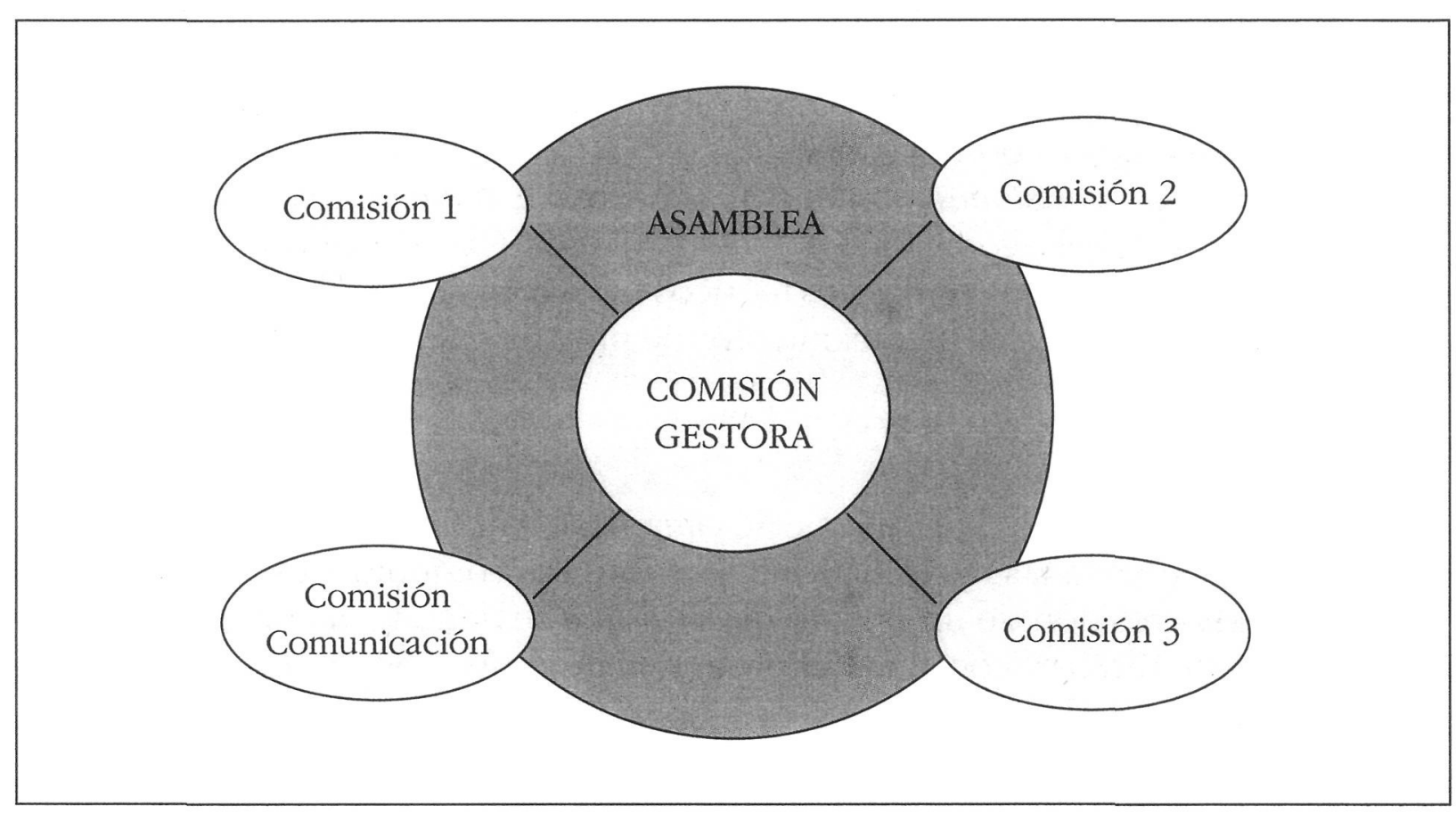


Las comunidades de aprendizaje plantean grandes retos, estableciendo concreciones educativas para potenciar el proceso de innovación educativa y la participación de los distintos agentes de la comunidad en los espacios de decisión. En las comunidades de aprendizaje se deberán plantear estos espacios de decisión, transformando las estructuras organizativas tradicionales de la escuela en estructuras de decisión más democráticas. Las comisiones mixtas de trabajo son espacios de decisión importantes y democráticos que existen en una comunidad de aprendizaje, cuya función es organizar y gestionar los distintos proyectos del centro educativo, así como intentar conseguir los objetivos y las actuaciones que se planifiquen y se implementen desde cada uno de ellos.

Las funciones más importantes de la comisión gestora son:

- Comunicación entre las comisiones mixtas de trabajo existentes.

- Dar respuesta a posibles problemas.

- Agilizar la coordinación entre las comisiones.

La comisión gestora coordina las diferentes comisiones para que no se repitan los distintos proyectos y para optimizar el trabajo. En esta comisión gestora existen representantes de todas las comisiones, del equipo directivo y del claustro.

\section{b) Los recursos}

La adquisición y distribución de los recursos materiales, humanos y funcionales en las comunidades de aprendizaje se basan en dos acciones organizativas básicas: la apertura a la comunidad y el trabajo por comisiones ${ }^{19}$. El centro educativo deberá abrirse a su entorno con acciones como la ampliación del horario de apertura del centro educativo, la oferta de biblioteca, los campos de deporte, el gimnasio, el aula de informática a la gente del barrio, y la oferta de espacios a otros centros educativos e instituciones, etc. De esta forma se conseguirán atraer muchos recursos potenciales, como pueden ser el voluntariado, la intervención de entidades de educación no formal, la captación de recursos a través de empresas cercanas a la comunidad, etc. Cada comisión establecerá sus mecanismos de captación de los recursos necesarios para llevar a cabo sus proyectos. También se podrá crear una comisión exclusiva de búsqueda de recursos. Así se puede decir que:

El objetivo del desarrollo comunitario disminuirá su carácter de servicio público a cargo de la administración, inclinándose hacia la autocreación y autogestión de los propios recursos por parte de la comunidad [...]. Reducirá las iniciativas directamente

19. El trabajo por comisiones establecerá equipos de trabajo dedicados exclusivamente a la adquisición de recursos de todo tipo. La demanda de recursos no se deberá centralizar en el equipo directivo, se deberá extender el radio de acción y de captación. Cada comisión establecerá los mecanismos de captación de los nuevos recursos necesarios para llevar a cabo sus proyectos. 
gestionadas por la administración y aumentará su política de subvenciones a las creadas en su periferia o completamente al margen del mismo (Flecha, 1990, 78).

En definitiva, se trata de que el centro educativo pueda explotar todos los recursos y todas las posibilidades socioeducativas que presenta, abriéndose a su entorno y haciendo que éste se familiarice con el mismo.

\subsection{Los planteamientos institucionales}

La participación en el centro educativo de todos los agentes implicados es fundamental, así se recoge que "la expansión actual responde a la necesidad de intensificar la socialización participativa de las personas adultas. Por un lado, se pretende así lograr más que un simple control social, se aspira desde muchas instituciones a conseguir la adhesión activa de los ciudadanos a su proyecto" (Flecha, 1990, 89).

El fruto de la máxima participación de las personas que integran la comunidad de aprendizaje está reflejado en el PEC y en el PCC. En la elaboración de estos documentos oficiales del centro, el claustro de profesores y el equipo directivo son parte fundamental, participan de una forma especial; el resto de agentes dejan de ser representados por el consejo escolar y pasan a formar parte del proceso de forma directa a través de la asamblea y de las comisiones de trabajo. Una peculiaridad con respecto al PCC es que el diagnóstico inicial del centro educativo deja de recaer en exclusiva sobre el equipo directivo y el claustro de profesores, y pasa por la priorización de necesidades que se establecen en la asamblea. El resto de documentos oficiales del centro, el RRI, el DOC y la PGA, son consecuencia directa de los documentos anteriores. La Memoria Final del curso se realizará en función de la evaluación de las actuaciones realizadas por las comisiones durante todo el curso y la exposición de propuestas de mejora para el curso escolar siguiente en el marco de la asamblea. La participación de los agentes implicados en la comunidad educativa puede plantear una serie de cuestiones como por ejemplo la complejidad de las relaciones, la legitimidad de las acciones, la potestad para participar en la toma de decisiones, la posible confusión de roles, el temor a la pérdida de rigor profesional, etc. Para responder a este tipo de cuestiones se requiere un planteamiento de base que oriente esta nueva forma de entender las relaciones.

La transformación de la escuela en comunidad de aprendizaje plantea la unión de escuela y entorno como agentes educativos y, por tanto, el trabajo conjunto, pero para que éste se pueda llevar a cabo con unas mínimas garantías de éxito, se tienen que poner en marcha los siguientes mecanismos (Martínez, 2004, 17):

- Cambiar la mentalidad de recepción de un servicio público por la mentalidad de protagonismo en la función pública.

- Cambiar las pretensiones de poder por las pretensiones de validez en el discurso de los agentes de la comunidad de aprendizaje utilizando planteamientos dialógicos. 
El planteamiento organizacional de una comunidad de aprendizaje respecto de un centro educativo ordinario, se diferencia fundamentalmente en el grado de participación de todos los agentes de la comunidad en el centro y en el grado de implicación en los procesos de toma de decisiones. El objetivo común a todos los colectivos que trabajan en los procesos de enseñanza-aprendizaje es la excelencia educativa de los alumnos y la transformación social de la comunidad.

\section{INTERACCIONES COMO BASE EN LAS COMUNIDADES DE APRENDIZAJE: LOS GRUPOS INTERACTIVOS}

En las comunidades de aprendizaje se parte de la idea de que todas las personas tienen capacidad de transformación ${ }^{20}$, a través del diálogo intersubjetivo es posible dar un nuevo sentido a la vida de las personas implicadas, creando los canales necesarios para superar situaciones de desigualdad y exclusión, mediante la formación, la cooperación y las decisiones por consenso de todos sus miembros.

Las comunidades de aprendizaje se convierten en un proyecto comunitario de formación, en el que los profesionales de la educación dejan de tener todo el poder de decisión sobre la mejor educación para los niños y las niñas, y pasan a cooperar de forma entusiasta con otros agentes educativos de la comunidad para maximizar los aprendizajes de todos y todas. Con este mismo objetivo, la oferta educativa se amplía a toda la comunidad, las puertas del centro se abren a la formación de las familias en respuesta a sus propias demandas formativas (Elboj, $2002,95)$.

El aprendizaje dialógico fomenta el diálogo reflexivo en todos los ámbitos educativos, tanto en el aula como fuera de ella. Una de las formas de desarrollar ese diálogo reflexivo es a través de los grupos interactivos.

\subsection{Definición y características de los grupos interactivos}

La historia interactiva en grupos como son la familia y la pandilla nos ha proporcionado gran parte de la esencia humana como seres sociales, con la participación en grupos de trabajo se actualizan nuestras vivencias grupales primarias y se pueden descubrir muchas pautas de intervención. En los grupos, los participantes establecen contactos sociales primarios y desarrollan su yo personal, además de potenciar la socialización primaria y la integración social.

El grupo es un sistema formado por personas que interactúan, no es una mera suma de sus miembros, posee entidad y características propias diferentes de las de

20. El contenido transformador del aprendizaje dialógico tiene diversas implicaciones y fundamentos. Una dimensión ética personal, recuerdan las palabras de FreIre (1997) en Pedagogía de la autonomía. 
sus miembros, produciendo una sinergia de las experiencias y conocimientos de éstos. Cada miembro del grupo se relaciona con los demás de tal forma que un cambio en uno de ellos supone un cambio en la totalidad. Los miembros de cada grupo interactúan en el seno del grupo, así como los diferentes grupos mixtos interactúan entre ellos y con el contexto exterior. Los grupos interactivos son una organización flexible del aula.

Una de las principales ventajas del trabajo en grupo es el aprendizaje de habilidades sociales en la interacción personal y activa, además del aprendizaje mismo de las prácticas cooperativas y de aparecer fenómenos de comunicación, liderazgo, toma de decisiones, manejo de conflictos y procesamiento del grupo. La estructura cooperativa favorece la motivación, la autoestima y el área emocional y social de las personas. Los resultados experimentales muestran la superioridad del aprendizaje cooperativo, siendo superior el logro, la empatía, la autoestima y la salud psicológica de los participantes del grupo (Johnson, 1980). El trabajo en grupo exige un talante especial y una organización funcional en el grupo para que todos los miembros participen y sientan realmente su rol en el grupo lo que permite la consecución de los objetivos, retroalimentándose de las interacciones con los demás miembros.

Los grupos interactivos son grupos heterogéneos formados por pocos alumnos que mantiene el nivel de motivación y atención. Las actividades realizadas en cada grupo están tutorizadas por una persona adulta, voluntarios de la comunidad, que, en numerosas ocasiones, son familiares. A través de grupos interactivos heterogéneos ${ }^{21}$ se refuerza el aprendizaje de todos los niños y niñas sin ningún tipo de discriminaciones, superando las soluciones parciales que a través de la creación de aulas especiales o unidades externas se pretenden dar a niños y niñas considerados problemáticos, conflictivos, lentos, o con dificultades de aprendizaje (Puigdellivol, 1998).

El trabajo en grupos interactivos potencia valores como la solidaridad entre las diferentes culturas, pero también el aprendizaje instrumental. La dinámica que se genera en el grupo asegura que todos los participantes se sientan responsables del propio aprendizaje, así como del aprendizaje del resto de los compañeros.

La metodología y la organización del trabajo en grupo es una buena estrategia para implementar el proyecto de las comunidades de aprendizaje y para poder conseguir los objetivos propuestos. Así el grupo de clase se puede dividir en pequeños grupos de trabajo, modificando la estructura espacial tradicional del aula. Cada grupo estará tutorizado por un apersona adulta (voluntarios, familiares, antiguos alumnos, etc.), facilitando nuevos espacios de trabajo orientados a la aceleración del aprendizaje comunicativo y cooperativo mediante un seguimiento

21. Para profundizar en la formación, organización, funcionamiento y estructura de los grupos interactivos heterogéneos, se puede consultar FlECHA, Ramón (2002) Comunidades de aprendizaje. Transformar la educación, 93. 
individualizado y grupal. Respecto a los objetivos a conseguir en el pequeño grupo, se puede decir que "el rasgo distintivo para conocer si un pequeño grupo está realizando un trabajo cooperativo no es su tamaño, sino si los objetivos de los participantes se hallan vinculados de tal modo que cada cual sólo pueda alcanzar sus objetivos sí, y sólo sí, los demás consiguen los suyos" (Rué, 1989).

Los grupos de trabajo interactivos plantean objetivos comunes, así "un grupo social consta de un determinado número de miembros, quienes para alcanzar un objetivo común (objetivo del grupo), se inscriben durante un tiempo prolongado en un proceso relativamente continuo de comunicación e interacción y desarrollan un sentimiento de solidaridad (sentimiento de "nosotros")" (Schafers, 1984, 26). Respecto a las estrategias de aprendizaje, la metodología de los grupos interactivos es una "estrategia que se desarrolla dentro de las aulas para acelerar el aprendizaje, aceleración que se produce mediante la aplicación de metodologías didácticas que catalizan y estimulan distintas condiciones y entornos educativos que inciden de forma positiva en el aprendizaje del alumno" (Ferrer Esteban, 2004, 21).

Desde la perspectiva de aceleración del aprendizaje se definen los grupos interactivos y se caracterizan como una estrategia metodológica eficaz.

La aceleración del aprendizaje se entiende como una medida pedagógica que responde a criterios de justicia y equidad en un marco de producción y reproducción de desigualdades sociales y educativas (Fòrum IDEA, 2002). Las estrategias metodológicas planteadas desde el proyecto "Comunidades de aprendizaje", apuntan al enriquecimiento de los procesos de enseñanza-aprendizaje, tratando de impulsar y acelerar el aprendizaje del alumnado mediante la superación de las estructuras escolares tradicionales y la participación de toda la comunidad educativa (Elboj, 2002; Fòrum IDEA, 2002).

En el marco del desarrollo de las comunidades de aprendizaje, se produce la aceleración del aprendizaje (Levin, 1987; Bonal, 1992), mediante la aplicación de metodologías didácticas que catalizan y estimulan distintas condiciones y entornos educativos que inciden de forma positiva en el aprendizaje del alumno.

Los grupos interactivos son una estrategia didáctica activa que pretende conseguir los siguientes objetivos (CREA, 2002):

- Desarrollar operaciones cognitivas elementales: memoria, atención, etc.

- Aprender reglas prácticas heurísticas para la ejecución de un dominio de tareas.

- Mostrar mecanismos de pensamiento empleados en la resolución de problemas.

- Desarrollar procesos de aprendizaje cooperativo y dialógico. 


\subsection{El aprendizaje dialógico}

La concepción comunicativa ${ }^{22}$ plantea que el aprendizaje depende principalmente de las interacciones entre personas. En estas interacciones se produce un diálogo intersubjetivo que se desarrolla de forma democrática y horizontal, y todas las personas tienen las mismas posibilidades de intervenir y de actuar, como medio de acción común y de superación de desigualdades.

El lenguaje dialógico no es sólo una construcción mental más o menos verosímil sino que, en unión de otras medidas organizativas y participativas, es especialmente una forma de favorecer los aprendizajes, especialmente importante en contextos socialmente desfavorecidos en los que otras soluciones tienen resultados parciales. El aprendizaje dialógico es válido en contextos educativos que van desde la infancia hasta la madurez, tal como se está haciendo ya en el presente. Se ha llevado a la práctica en experiencias globales como comunidades de aprendizaje en centros escolares de País Vasco, Cataluña y Aragón, y en actividades concretas de educación de personas adultas como las tertulias literarias (Flecha, 2002, 92).

El proyecto de transformación educativa de las comunidades de aprendizaje en el que están contextualizados los grupos interactivos, se fundamenta en varios principios, uno de ellos es el aprendizaje dialógico (Flecha, 1997; Elboj, 2002; Sánchez, 1995), que es una concepción comunicativa del aprendizaje que sostiene que éste depende principalmente de las interacciones entre las personas (Casamitjana $\mathrm{y}$ otros, 2000).

Un diálogo es igualitario cuando se valoran las aportaciones de cada participante en función de los argumentos de validez que se exponen. En la sociedad actual de la información y la comunicación, en la que los aprendizajes realizados en los contextos no formales e informales cobran importancia, es posible un diálogo en el que cada persona puede aportar su conocimiento y experiencia desarrollada en muy diversos ámbitos ${ }^{23}$. El consenso se encontrará en la aceptación común de los mejores argumentos, mientras que el diseño hace posible la búsqueda de mejores razones para sustentar las posiciones.

22. En la teoría de la acción comunitaria, se desarrolla una concepción de la competencia comunicativa en la que se demuestra que todas las personas son sujetos capaces de lenguaje y acción (HABERMAS, 1987). Con la teoría de la modernización reflexiva, se demuestra que la reflexión mediante el diálogo es un elemento cada vez más determinante en nuestra sociedad y de nuestra individualidad (BECK, 1998). Se puede demostrar que el diálogo reflexivo provoca más aprendizaje instrumental y más solidaridad que la enseñanza tradicional o la individualización del proceso (FreIRE, 1997a; CREA, 1999).

23. El diálogo, como intercambio de análisis y razones, es fundamental para el avance del conocimiento y de la curiosidad epistemológica. Así Freire señala que "La experiencia dialógica es fundamental para la construcción de la curiosidad epistemológica. Son elementos constitutivos de ésta: la postura crítica que el diálogo implica, su preocupación por aprender y la razón de ser del objeto que media entre los sujetos del diálogon (FreIre, 1997b, 110-111). 


\subsection{La organización de los grupos interactivos}

Para el buen desarrollo y aplicación de los grupos interactivos en el aula, se deben tener en cuenta los siguientes aspectos organizativos:

a) Pequeño grupo. El gran grupo de clase se dividirá en pequeños grupos de trabajo interactivos.

b) Composición de los grupos. La composición de los grupos debe ser flexible y heterogénea.

c) Autorización. En cada grupo interactivo hay una persona adulta (profesor o voluntario) que realiza tareas de autorización.

d) Distribución de tiempos y de alumnos ${ }^{24}$. Dependiendo de los diferentes grupos interactivos, a cada grupo se le asignarán los tiempos adecuados, para la realización de las distintas actividades; y el número de alumnos recomendado.

e) Contenidos. El tema curricular se trabajará mediante distintas actividades desde cada grupo interactivo. Cada actividad contempla un tema coherente con el tema principal y complementario con los tratados en los otros grupos. Cada grupo tiene una secuencia didáctica autónoma y debe man-. tener una relación epistemológica.

f) Contextualización de las actividades. Al comienzo y al final de la clase, es conveniente que el profesor sitúe las actividades en un mismo marco temático. Al comienzo, introduciendo el tema general, y al finalizar, aportando conclusiones a todas las actividades realizadas.

\section{CONCLUSIÓN}

Las experiencias de los proyectos de las comunidades de aprendizaje, tanto en el ámbito internacional como nacional, han sido experiencias que han alcanzado éxito en los centros educativos en los que se han desarrollado, consiguiendo una enseñanza de calidad. Las comunidades de aprendizaje desarrollan una educación teórica y práctica basada en el análisis, la reflexión, el diálogo, la igualdad de oportunidades, la transformación, etc., convirtiendo al alumno en protagonista de su propio aprendizaje.

Con el desarrollo de las comunidades de aprendizaje en los centros educativos se podrán paliar diferentes aspectos negativos de la educación como el fracaso escolar, el absentismo, la falta de motivación escolar, la precariedad económica, las situaciones de riesgo y más desfavorecidas, etc.; y mejorar el nivel académico y cultural

24. "Dependiendo del ciclo en el que se desarrolle la metodología de los grupos interactivos, partimos de criterios distintos con relación con el tiempo dedicado a cada actividad y con el número recomendado de alumnos por grupo. Con relación a la variable "tiempo", también se considera que el tiempo total de la clase se debe dividir aproximadamente por el número de grupos creados. Por tanto, la rotación del alumno se producirá al finalizar el tiempo destinado a cada actividad. (FerRer, 110-111). 
de los alumnos, erradicando la exclusión. Asimismo se favorece la participación, la formación, la investigación, la convivencia y la solidaridad de los diferentes agentes educativos.

Las comunidades de aprendizaje se organizan por comisiones de trabajo que tratan de planificar, gestionar y desarrollar los distintos proyectos, estos proyectos responden a las necesidades detectadas en los centros educativos. También utilizan los grupos interactivos como estrategias eficaces para potenciar la motivación, el diálogo y la participación, y conseguir la aceleración del aprendizaje.

Para la incorporación progresiva de los ciudadanos a la sociedad del conocimiento, es necesario que los centros educativos amplíen sus redes y procesos hasta desarrollar auténticas comunidades de aprendizaje, en las que participen todos los colectivos de la comunidad educativa (profesorado, alumnado, familias, asociaciones y demás instituciones implicadas en la educación). Los centros educativos se deberán relacionar con su entorno, modificando el sistema de participación comunitario, las funciones del profesorado y las relaciones entre los diferentes colectivos de la comunidad educativa. Las cuestiones educativas que se abordarán en un centro educativo en el que se desarrolle una comunidad de aprendizaje son muy diferentes a las abordadas por fórmulas tradicionales. Por tanto, partiendo de los resultados eficaces que se obtienen en la enseñanza con el desarrollo de las comunidades de aprendizaje en los centros educativos, se propone:

- Transformar los centros educativos en verdaderas comunidades de aprendizaje. Para conseguir esta transformación, en primer lugar, es necesario cambiar las estructuras internas de los centros educativos, las relaciones existentes entre los diferentes colectivos y también las relaciones existentes con su entorno. En segundo lugar, consensuar las propuestas por los diferentes colectivos de la comunidad y planificar los proyectos correspondientes. En estos proyectos se deberán implicar todos los agentes de la comunidad para conseguir un cambio importante en la comunidad educativa.

- Potenciar la participación y la colaboración de todo el claustro de profesores del centro en el proyecto de comunidades de aprendizaje, para que así todos los profesores se impliquen en el proyecto, adquiriendo un alto grado de compromiso y responsabilidad. Recoger las demandas del profesorado dando respuesta a través de la formación y asesoramiento en los programas necesarios. Una vez que se ha formado un equipo de profesores comprometidos con el proyecto, dar continuidad a este equipo manteniéndolo estable durante los cursos siguientes.

- Implicar al alumnado en la participación del proyecto de las comunidades de aprendizaje. Favoreciendo su aprendizaje con la organización de refuerzos en el aula y conseguir las competencias básicas necesarias.

- Motivar a los padres y madres para que colaboren en los centros educativos, participando en el desarrollo del proyecto de comunidades de aprendizaje. Así se podrá favorecer la integración de las familias en el 
centro educativo, dando respuestas a sus inquietudes, concretando sus demandas y creando espacios de formación.

- Tener una visión más crítica y amplia que permita avanzar hacia la toma de decisiones cada vez más adaptadas a la realidad educativa de cada centro educativo.

- Utilizar y aplicar las nuevas tecnologías de la información y la comunicación en la comunidad educativa, debido a que son imprescindibles en la sociedad actual.

En definitiva, se pretende mejorar la calidad de la enseñanza en los centros educativos con la implementación de las comunidades de aprendizaje, formando íntegramente a los alumnos y adaptándolos a la sociedad del conocimiento, para que puedan superar los retos que esta sociedad les plantea. Por tanto, el desarrollo de las comunidades de aprendizaje implica una transformación educativa y social en los centros educativos, mediante la cual se educará a los ciudadanos del siglo XXI.

\section{BiBLIOGRAFÍA}

ANDER-EGG, E. (1981) Metodología y práctica de la animación sociocultural. Madrid, Editorial Marsiega.

Apple, M. W. y Beana, J. A. (1997) Escuelas democráticas. Madrid, Morata.

BECK, U. (1998) La sociedad del riesgo: hacia una nueva modernidad. Barcelona, Paidós.

BeCK-Gernsheim, E.; ButLer, J. y PuigverT, L. (2003) Woman \& Social Transformation. New York, Peter Lang.

Bonal, X. (1992) Escuelas aceleradas para alumnos desaventajados, Cuadernos de Pedagogia, 201, 60-65.

CASAmitJana, M.; Puigvert, L.; SOler, M. y TORTajada, I. (2000) Investigar y transformar: CREA Centro de Investigación Social y Educativa, Cultura y Educación, 17/18, 117-129.

Colom, A. J.; Pérez, P. M. y VÁzquez, G. (2001) Calidad de vida, ambiente y educación, en VÁzQuez. G. (ed.). Educación y calidad de vida. Madrid, Editorial Complutense, 1-37.

Comunidades de aprendizaje: participación, calidad y transformación social, Educar, 29, 103-121.

CREA (1999) Cambio Educativo y Social I. Teorias y Prácticas que superan desigualdades. Jornadas celebradas el 22 y 23 de noviembre en el Parc Cientific de Barcelona.

- (2002) Comunidades de aprendizaje. Documento policopiado. Universitat de Barcelona.

DELORS, J. y otros (1996) La educación encierra un tesoro. Informe a la UNESCO de la Comisión Internacional sobre educación para el siglo XXI. Madrid, Santillana/UNESCO.

ElBoj, C.; Puigdellivol, I.; SOlER, M. y VAlls, R. (2002) Comunidades de aprendizaje. Transformar la educación. Barcelona, Graó.

Ely, D. P. (1992) Tecnología educativa: campo de estudio, en Husen, T. y PostLeTMwaITe, T. N. Enciclopedia Internacional de la Educación, 9, 5394-5397.

Ferrer Esteban, G. (2004a) Experiencia: Las comisiones mixtas de trabajo en las comunidades de aprendizaje, Temáticos Escuela, 11, 18-20.

- (2004b) Estrategias e instrumentos. Los grupos interactivos, Temáticos Escuela, 11, 21-24. 
FLECHA, R. (1990) La nueva desigualdad cultural. Esplugues del Llobregat, El Roure.

- (1997) Compartiendo palabras. Barcelona, Paidós.

FÒRUM IDEA (2002).

Freire. P. (1977) Cambio. Bogotá, Ediciones América Latina.

- (1992) Pedagogía de la esperanza. Barcelona, Paidós.

- (1997a) A la sombra de este árbol. Barcelona, El Roure.

- (1997b) Pedagogía de la autonomía. Madrid, Siglo XXI.

GRUNDY, S. (1987) Vitae: product o praxis. London, The Falmer Press.

Habermas, J. (1987) Teoría de la acción comunitaria, vol. I. Madrid, Taurus.

Jaussi, M. L. (2003) Comunidades de aprendizaje. Vitoria, Publicaciones Gobierno Vasco.

JAusSI, M. L. y LunA, F. (2002) Comunidades de aprendizaje. Una experiencia de participación de toda la comunidad educativa para mejorar la calidad de la enseñanza, Cuadernos de Pedagogia, 316, 39-67.

- (1998) C.P. Ramón Bajo de Vitoria-Gasteiz. Una comunidad de aprendizaje, Cuadernos de Pedagogia, 270, 36-44.

JoHnson, D. W. (1980) The effects of cooperative, competitive, and individualistic conditions on students problem solving performance, American Educational Research Journal, $17,83-94$.

LEVIN, M. H. (1987) New Schools for the Disadvantaged, Teacher Education Quarterly, 14 (4), 60-83.

- (1998) Accelerated Schools: A Decade of Evolution, en Hargreaves, A.; Lieberman, A.; FULLAN, M. y HOPKINS, D. (eds.). International handbook of Educational chanse (II). Boston, Kluwer Academic Publismers; 807-830.

MALLAS, S. (1979) Medios audiovisuales y pedagogía activa. Barcelona, CEAC.

MARTíNez, M. (2004a) El contexto de las comunidades de aprendizaje, Temáticos Escuela, 11, 4-6.

- (2004b) Bases teóricas de las comunidades de aprendizaje, Temáticos Escuela, 11, 7-10.

- (2004c) El desarrollo de una comunidad de aprendizaje, Temáticos Escuela, 11, 11-14.

Martínez Ortiz, S. (2004) Aspectos organizativos de una comunidad de aprendizaje, Temáticos Escuela, 11, 15-17.

McCuntock, R. O.; STReiBel, M. J. y VázQuez Gómez, G. (1993) Comunicación, Tecnología y Diseños de instrucción: La construcción del conocimiento escolar y el uso de los ordenadores. Madrid, MEC.

Monroe, W. S. (1941) Enciclopedia of Educational Research. New York, MacMillan.

NATRIEllo, Gary; McDill, Edward M. y Pallas, A. M. (1990) Schooling Disadvantaged children: Racing Against Catastrophe. New Cork, Teacher College.

PuigdeILIVOL, I. (1998) La educación especial en la escuela integrada: una perspectiva desde la diversidad. Barcelona, Graó.

RuÉ, J. (1989) El trabajo cooperativo por grupo, Cuadernos de Pedagogia, 170, 18-21.

SÁnCHEZ Aroca, M. (1999) La Verneda Sant Martí: a schools where people tareto dream, Harvard Educational Review, 69 (3), 320-335.

SCHAFERS, B. (1984) Los grupos primarios. Barcelona, Herder.

SLAVIN, R. E. y otros (1996) Every child, every school: success for all. Thousand Oaks, California, Corwin Press.

Trilla, J.; Ayuste, A.; Romaña, T. y Salinas, M. (2001) Educación y calidad de vida. Las cosas, los otros y uno mismo, en VÁzQuez, G. (ed.). Educación y calidad de vida. Madrid, Editorial Complutense, 117-167.

UNED (1993) Tecnología educativa. Materiales audiográficos. Madrid, UNED. 